\title{
Los shibolets del procés: Vilajosana sobre el derecho a decidir
}

\section{The shibolets of the procés: Vilajosana on the right to decide}

\author{
José Juan Moreso* \\ Universitat Pompeu Fabra \\ ORCID ID 0000-0003-2702-569X \\ josejuan.moreso@upf.edu
}

\section{Cita recomendada:}

Moreso, J. J. (2020). Los shibolets del procés: Vilajosana sobre el derecho a decidir. Eunomía. Revista en Cultura de la Legalidad, 19, pp. 495-505.

\section{Resumen}

En este comentario se discute la articulada defensa de Vilajosana (2020) de un derecho a decidir la secesión de España por parte de los ciudadanos de Cataluña. Se argumenta que dicho derecho, entendido como un derecho (claim) que comporta deberes correlativos, no es legal conforme a la Constitución española, puesto que lo máximo que esta concede es un derecho (liberty), que está sometido a las exigencias que la Constitución dispone. Se arguye también que dicho derecho no es legítimo, desde el punto de vista de la filosofía política, porque no hay ningún modo conceptualmente plausible de atribuir dicho derecho a un subconjunto de ciudadanos de un Estado y no a otro, incluido el subconjunto formado por todos los ciudadanos del Estado, dado que todo conjunto es un subconjunto de sí mismo.

\section{Palabras clave}

Vilajosana, Cataluña, secesión, derecho a decidir, estrategia plebiscitaria.

\begin{abstract}
In this comment, the articulated defence by Vilajosana (2020) of a right to decide of the Catalan citizens for seceding from Spain is discussed. It is argued that such right, understood as a claim-right, which involves correlative duties, is not legal in accordance with the Spanish Constitution, because at most the Spanish Constitution authorize a liberty-right, subjected to the stipulated requirements by the Constitution. It is also defended that such right is not legitimate, from the political philosophy point of view, because there is no conceptually plausible way to attribute the right to a subset of citizens in one State and not to other subset, including the subset integrated by all the citizens of the State, given that every set is a subset of itself.
\end{abstract}

* Catedrático de Filosofía del Derecho. Departamento de Derecho. Universitat Pompeu Fabra (Barcelona). 


\section{Keywords}

Vilajosana, Catalonia, secession, right to decide, plebiscitary strategy.

SUMARIO. 1. Introducción. 2. Un espacio para el acuerdo. 3. ¿Un derecho constitucional a decidir? 4. La legitimidad y la objeción Matrioska. 5. A modo de conclusión: el poder y el derecho

Occupaveruntque Galaaditæ vada Jordanis, per quæ Ephraim reversurus erat. Cumque venisset ad ea de Ephraim numero, fugiens, atque dixisset: Obsecro ut me transire permittatis: dicebant ei Galaaditæ: Numquid Ephrathæus es? quo dicente: Non sum : interrogabant eum : Dic ergo Scibboleth, quod interpretatur Spica. Qui respondebat: Sibboleth : eadem littera spicam exprimere non valens. Statimque apprehensum jugulabant in ipso Jordanis transitu. Et ceciderunt in illo tempore de Ephraim quadraginta duo millia.

(Liber ludicum, 12, 5-6, La Biblia Vugata)

Los Galaaditas tomaron los vados del Jordán á Ephraim; y era que, cuando alguno de los de Ephraim que había huído, decía, ¿pasaré? Los de Galaad le preguntaba: ¿eres tú Ephrateo?

Si él respondía, No; entonces le decían: Ahora pues, di, Shiboleth. Y él decía, Siboleth; Porque no podía pronunciar de aquella suerte. Entonces le echaban mano, y le degollaban junto a los vados del Jordán. Y murieron entonces de los de Ephraim cuarenta y dos mil.

(Libro de los Jueces, 12, 5-6, La Biblia Reina Valera (Biblia del Cántaro))

\section{Introducción}

He sido convocado, debido a la proverbial amabilidad del director de esta Revista, mi amigo José María Sauca, a participar en este debate comentando la relevante contribución de Vilajosana (2020) a pergeñar unos fundamentos para el denominado «derecho a decidir», en el contexto del proceso catalán. Josep M. Vilajosana es también un colega en la misma universidad, y un amigo.

No es la primera vez que Vilajosana se ocupa de esta cuestión (véase Vilajosana, 2014a, 2014b y 2019), pero en este texto consigue destilar sus tesis con meridiana claridad y de un modo especialmente perspicuo. Lo que facilita mucho la tarea de delimitar los acuerdos y, también, los desacuerdos.

Los acuerdos guardan relación con lo que podemos denominar la «doctrina canadiense», referida a la opinión consultiva de la Corte Suprema del Canadá (Reference Re Secession of Quebec [1998] 2 S.C.R $)^{1}$, con arreglo a la cual el principio democrático, en un contexto federal, requiere al gobierno de la Federación una negociación de buena fe con una provincia, el Québec en su caso, cuya población ha mostrado amplia y democráticamente la voluntad de separarse, una negociación para lograr una solución consensuada.

\footnotetext{
${ }^{1}$ En Moreso (2018) traté de aplicar dicha doctrina al caso catalán.
} 

afirma:

Los desacuerdos tienen que ver con lo siguiente, Vilajosana (2020, p. 377)

Hay quien sostiene que las reivindicaciones provenientes de los partidarios del llamado «derecho a decidir» ni tienen cobertura legal ni son legítimas; los hay que consideran que, a pesar de que sean legítimas, no están amparadas por la legalidad; alguno habrá que entienda que, aunque fueran legales, carecerían de legitimidad; $y$, por último, hay quien opina que estas pretensiones pueden considerarse no únicamente legítimas, sino también ajustadas a la legalidad.

Y, enfáticamente, añade: «En lo que sigue voy a defender esta última opción». Pues bien, si el derecho a decidir se entiende, como creo que lo entiende Vilajosana, como un derecho-pretensión -un claim-a un referéndum vinculante sobre la secesión de Cataluña, entonces creo que dicho derecho no es -insisto, en el sentido de un claim- ni legal, de acuerdo con la constitución española, ni legítimo con arreglo a la filosofía política más admisible.

Procederé del siguiente modo: en la sección segunda, trataré de hacer más explícitos mis acuerdos con Vilajosana. En la sección tercera, intentaré argumentar por qué no hay un derecho como claim en la constitución española. En la sección cuarta presentaré los argumentos contra la legitimidad de dicho derecho. $Y$ en la sección quinta concluiré.

Pero antes de ello, deseo hacer la siguiente advertencia, ex abundante cautela. A menudo, en este debate (también Vilajosana, 2020, p. 385, nota 17) se hace referencia a los referéndums del Québec (1980 y 1995) y al de Escocia (en 2014), argumentando que las democracias canadiense y británica, respectivamente, autorizaron, o al menos toleraron, la celebración de un referéndum a lo que, hasta ahora, el gobierno de España siempre se ha negado. Esto es verdad. Pero también lo es, y esto se suele ocultar, que ni en Québec ni en Escocia sus Parlamentos y sus gobiernos se embarcaron en un proceso unilateral hacia la secesión. Recordemos los hechos: el día 1 de octubre de 2017 se celebró el referéndum para decidir acerca de la independencia de Cataluña -con la pregunta "¿Quiere que Cataluña sea un Estado independiente en forma de República?»-, convocado en virtud de una Ley aprobada por el Parlamento de esta comunidad autónoma, a la que se suma la denominada Ley de transitoriedad que, entre otras cosas, establece que si en la consulta resulta haber más respuestas afirmativas que negativas, el Parlament declarará la independencia de Cataluña ${ }^{2}$. Ambas leyes, como es sabido, fueron suspendidas cautelarmente por el Tribunal Constitucional, como consecuencia de la aceptación a trámite de sendos recursos del Gobierno de España y, en 2017, declaradas nulas por el Tribunal Constitucional ${ }^{3}$. Es cierto que la aprobación de ambas leyes fue un acto de suprema deslealtad constitucional. Aprobaron las dos leyes más relevantes de la historia de la Generalitat contemporánea, en sesiones en las que no aparecían previamente en el orden del día, ignorando el parecer de todos los letrados del Parlament, sin el preceptivo informe del Consell de Garanties Estatutàries, pretendiendo que dicho referéndum tiene, de modo unilateral, carácter vinculante. Ignoraron que, en una democracia constitucional, el derecho actúa como mediador de la política, de los objetivos políticos, y ello constituye el escudo de las libertades ciudadanas frente a

\footnotetext{
2 Llei 19/2017, del 6 de setembre, del referèndum d'autodeterminació i Llei 20/201, del 8 de setembre, de transitorietat jurídica i fundacional de la República.

${ }^{3}$ Autos 123 y 124 del Tribunal Constitucional, de 19 de septiembre de 2017 (BOE de 22 de septiembre). Ambas leyes declaradas nulas e inconstitucionales por las sentencias STC 114/2017, de 17 de octubre y STC 124/2017 de 8 de noviembre.
} 
las arbitrariedades del poder ${ }^{4}$. Decidieron ejercer el poder de modo arbitrario, el poder desnudo.

\section{Un espacio para el acuerdo}

Una de las tesis cruciales de Vilajosana (2020, p. 385) es la siguiente:

La concepción densa de la democracia, en definitiva, sirve para dotar de contenido a la interpretación evolutiva que esta institución requiere, tal como he explicado. Se sigue de todo lo que he sostenido en este apartado que la interpretación ponderada del principio democrático (concretado en el artículo 23.1) con el de la indisolubilidad es tal que permite (cuando menos, en sentido débil) la convocatoria de un referéndum sobre la independencia de Cataluña, pero veta una declaración unilateral. Ésta es una forma razonable de tomar estos principios como reglas ideales y definitorios del Estado democrático instaurado en el artículo 1.1 de la Constitución.

Estoy de acuerdo en que la interpretación restrictiva del texto constitucional, que sostiene que nunca un referéndum, aún consultivo ${ }^{5}$, acerca de la voluntad de los catalanes de seguir formando parte de España, es posible, dado que ello suplantaría el proceso de reforma constitucional, es contraria a una consideración, que comparto, de la Constitución como un «árbol vivo» (vid., por todos, Waluchow, 2007). Vilajosana y yo estamos plenamente de acuerdo con la siguiente afirmación de la Corte Suprema del Canadá, en su ya famosa opinión consultiva (Reference Re Secession of Quebec [1998] 2 S.C.R., p. 273):

Resulta claramente de lo que precede que la secesión del Quebec del Canadá no puede ser considerada un acto legal si se lleva a cabo unilateralmente por la Asamblea nacional, la legislatura o el gobierno del Quebec, es decir sin previas negociaciones conforme a los principios. Cualquier proyecto de secesión de una provincia del Canadá que se lleve a cabo en desacuerdo con la Constitución del Canadá es también una violación del orden jurídico canadiense. Sin embargo, el orden constitucional canadiense no puede permanecer inmune en su existencia y su funcionamiento por la expresión no ambigua de una mayoría clara de quebequeses de no querer formar ya parte del Canadá. El principal medio de dar efecto a esta expresión es el deber constitucional de negociar conforme a los principios constitucionales que aquí hemos definido. Si las negociaciones se inician, nuestra Constitución, no menos que nuestra historia, apelarán a los participantes para esforzarse en reconciliar los derechos, las obligaciones y las aspiraciones legítimas de todos los canadienses en un marco que dará igual importancia a las responsabilidades y a los derechos constitucionales de cada uno.

Deseo advertir, sin embargo, que no me parece una estrategia adecuada la que usa Vilajosana para alcanzar dicha conclusión, consistente en una ponderación del principio democrático de nuestra Constitución con la indisolubilidad de España, establecida en el art. 2. No lo estoy porque creo que la indisolubilidad no es un principio, sino una de las reglas básicas de carácter "constitutivo» del ordenamiento español. La indisolubilidad no es ponderable. Podemos reformar la Constitución y establecer, con determinadas condiciones, el derecho a la secesión de uno de los

\footnotetext{
${ }^{4}$ En la mejor crónica jurídica del procés, un jurista íntegro -con simpatías nacionalistas declaradasnarra con estupor y tristeza estos hechos desde el privilegiado lugar de Letrado mayor del Parlament (Bayona, 2019).

${ }^{5}$ Esta, como es sabido, es la posición del Tribunal Constitucional, STC 103/2008, de 11 de septiembre y STC 42/2014, de 25 de marzo. Pero no hay acuerdo entre la doctrina constitucionalista. Véase una defensa autorizada de la posibilidad y, en ese momento, conveniencia de dicha consulta, en Rubio Llorente (2012), uno de los más prestigiosos constitucionalistas, anterior Vicepresidente del Tribunal, y también Queralt, Martí (2017). Para todo ello, Ferreres (2014, 2016), Fossas (2014), Tornos (2015).
} 
territorios que ahora integran España. La indisolubilidad tampoco es lo que los constitucionalistas denominan una cláusula «pétrea». En la Constitución española no hay cláusulas de tal naturaleza. La indisolubilidad es como la definición del tablero en el juego del ajedrez, si lo cambiamos, cambiamos de juego. Es semejante a la «monarquía parlamentaria» como forma de gobierno, no está sujeta a ponderación ninguna, podemos cambiarla mediante la reforma constitucional, pero no ponderarla con, por ejemplo, el principio democrático.

Esto no conlleva que el principio democrático no pueda desplegar los efectos que Vilajosana desea. Podemos considerar que el principio democrático exige al gobierno de España una negociación de buena fe, que incluya la posibilidad de convocar un referéndum. Si el referéndum acordado, en los términos que fuese, diese un resultado favorable a la secesión, entonces sólo se habría recorrido la mitad del camino -esta es la posición de la Corte Suprema del Canadá en la famosa opinión consultiva-. Quedaría todavía llevar a cabo un proceso de reforma constitucional, en los términos del art. 168 de la Carta magna, para alcanzar el final del trayecto.

\section{3. ¿Un derecho constitucional a decidir?}

Los partidarios del derecho a decidir (Barceló, 2016; López, 2017; y también, claro, Vilajosana, 2014a, 2020) se encargan de distinguir este derecho del derecho de autodeterminación de los pueblos reconocido por el derecho internacional, pero sólo -pues fue pensado para articular la descolonización- para supuestos de grave vulneración de los derechos humanos de un grupo cultural determinado o, también, para casos en los que un Estado ocupa militarmente el territorio de otro Estado y lo anexiona a su territorio, si es entendido como autodeterminación «externa», es decir, como derecho unilateral a la secesión (vd., por todos, la autorizada posición de Cassese, 1995). Vilajosana resume en tres las diferencias entre ambos derechos, de este perspicuo modo (2020, p. 381):

a. El derecho a decidir es un derecho individual-cuyos titulares son cada uno de los ciudadanos y ciudadanas de un determinado demos-, aunque se ejerza colectivamente; en cambio, el derecho a la autodeterminación es un derecho colectivo -cuyos titulares son los pueblos-.

b. Los derechos en cada caso se vinculan a dos diferentes formas típicas de ejercicio -aunque no necesariamente excluyentes-. En el primer caso, prima la realización de una consulta, que requiere alguna acción u omisión por parte del Estado -autorizándola, delegando su competencia o, simplemente, tolerándola-; en el segundo caso, se opta por la declaración unilateral de independencia.

c. La legalidad tomada como referencia en ambos casos es distinta. En el supuesto del derecho a decidir, sería el sistema jurídico estatal, mientras que en el caso del derecho a la autodeterminación sería el Derecho internacional.

Ahora bien, ¿qué tipo de derecho es el derecho a decidir? Como es sabido en teoría jurídica, 'derecho' es una expresión ambigua que se usa en muchos sentidos diversos. El más reconocido de los enfoques sobre los diversos sentidos que la expresión «derecho» tiene en el ámbito jurídico y, en general, en el ámbito práctico, es el de W.N. Hohfeld (1919). Hohfeld distingue cuatro sentidos diferentes de la expresión «derecho»: derecho como libertad - «liberty-right» o también «privilege-right»-, derecho como pretensión - «claim-right»-, derecho como potestad -«power-right»- y derecho como inmunidad -«immunity-right»-. Aquí nos interesan los dos primeros, en el primer sentido, en el sentido de derecho como libertad, "tener derecho a X»" significa algo como «hacer $X$ no está prohibido». En este sentido, por ejemplo, yo 
tengo el derecho como libertad a ir a cenar al Celler de Can Roca de Girona, tengo dicho derecho porque no hay norma alguna que me lo prohíba, ahora bien este derecho puede ser frustrado porque cuando trato de ir -como sucede a muchos a menudo- me dicen que no hay lugar ese día. En cambio, el segundo sentido de derecho, el derecho como una pretensión, comporta no sólo que me está permitido hacerlo, sino que los demás tienen el deber de no impedírmelo y, si se trata de una obligación a obtener algún servicio, que alguien tiene el deber de proporcionármelo. En este sentido, los derechos son correlativos a deberes. Así mi derecho a expresar mis ideas comporta que nadie puede impedírmelo y mi derecho a percibir mi salario que mi empleador tiene el deber de pagármelo ${ }^{6}$.

Vilajosana es bien consciente de esta distinción y se refiere a ella en otros términos, en los términos de von Wright (1963, p. 86. Vid. también Alchourrón-Bulygin, 1971, pp. 119-125) que distingue entre que un comportamiento esté permitido en sentido «débil», equivalente a la ausencia de una norma que lo prohíba y permitido en sentido «fuerte», es decir, un comportamiento que no está prohibido, pero está sometido a norma -es decir es o bien obligatorio o bien facultativo-. Y añade (Vilajosana, 2020, p. 381): «hay buenos argumentos para pensar que la Constitución permite, al menos en sentido débil, realizar una consulta a la ciudadanía de Cataluña sobre su futuro político».

Pero, obviamente, es muy relevante establecer si el derecho a realizar una consulta es un derecho como liberty o es un derecho como claim. Porque si es lo primero entonces la ciudadanía de Cataluña no dispone sin más de tal derecho para ejercerlo como desee. Es como si, por así decirlo, yo pretendiera que como no tengo prohibido cenar en el Celler de Can Roca, entonces sus propietarios tienen el deber de admitirme a cenar la noche en que lo solicito. Un formidable non sequitur, me temo. En cambio, si es lo segundo, entonces el gobierno de España tiene la obligación correlativa de autorizar la consulta.

El mismo Vilajosana (2020, p. 381) adecuadamente señala que se podría alcanzar una consulta de acuerdo con la constitución por dos vías:

En primer lugar, de manera directa, según lo dispuesto en el artículo 92. En este caso se permite que las decisiones políticas de especial transcendencia sean sometidas a referéndum, convocado por el Rey, mediante propuesta del gobierno [sic., el artículo dice «El presidente del gobierno»] y con la autorización del Congreso de los diputados. En segundo lugar, a tenor de lo dispuesto en el artículo 150.2, el Estado podría transferir o delegar la facultad de convocar un referéndum a la comunidad autónoma.

Pero esto precisamente muestra que el derecho de la ciudadanía catalana no es un claim-right, porque han de darse otras condiciones -la activación de uno de estos dos artículos constitucionales- para tener el derecho en el sentido fuerte. Ahora, en la situación actual, la ciudadanía catalana tiene, conforme a esta interpretación de la constitución, permitido sólo en sentido débil dicha consulta. Es decir, el derecho a decidir no es un derecho que pueda ejercerse de manera «unilateral». Quod erat demonstandum.

\section{La legitimidad y la objeción Matrioska}

Podría ser, es claro, que la constitución no reconociera un derecho unilateral a decidir, pero por razones normativas «debiera» reconocerlo, fuese «legítimo» que lo hiciera de este modo.

\footnotetext{
${ }^{6}$ La importancia para el tema que nos ocupa de esta distinción en Buchanan (2004, pp. 334-337 y 2017).
} 
En los últimos cuarenta años, en la literatura sobre la secesión se ha desarrollado una concepción, denominada «plebiscitaria», a veces también «democrática» (Beran, 1984, 1995; Philpott, 1995, Wellman, 1995). Esta es la posición que se halla, según creo, tras el denominado «derecho a decidir», y por eso vale la pena analizarla para averiguar si está en condiciones de legitimar un derecho unilateral a decidir.

La idea es muy simple: si una determinada mayoría de los ciudadanos de un territorio continuo que forma parte de un Estado, y dicho territorio es viable como nuevo Estado, tienen la voluntad de separarse del Estado matriz, entonces tienen el derecho -en el sentido de claim-right- de hacerlo.

Una de las ventajas que tiene esta posición, según sus defensores, es su simplicidad, sólo exige nuestra adhesión a los principios de autonomía de las personas y de democracia. De estos principios surge naturalmente el derecho de asociarse libremente con quien a uno le plazca.

Tiene, sin embargo, dificultades. Tiene dificultades de orden fáctico porque puede trastocar la estabilidad, ya frágil, del orden internacional y, también, de orden normativo, porque presupone la muy debatida doctrina del consentimiento como fundamento de la obligación política (vid., para todo ello, Buchanan 2004, pp. 373379).

No obstante, aquí voy a referirme a una dificultad más básica, una dificultad de carácter conceptual que denomino la objeción Matrioska, por razones que enseguida resultarán claras.

Supongamos que tenemos un conjunto $A$, cuyos miembros son: $\{v, w, x, y, z\}$ y hemos de decidir cómo generar subconjuntos a partir de él. Los miembros de $A$ son personas y pueden tomar decisiones. Para que un subconjunto de $A$ sea elegible es precisa la voluntad de la mitad más uno de sus miembros. Supongamos, entonces que $v$ y $w$ desean generar el subconjunto $B$, con los siguientes miembros $\{v, w, z\}$, pero $z$ no desea generar ese subconjunto. Entonces $B$ es elegible. Pero supongamos el subconjunto también posible $C:\{v, w, z, y\}$, en donde ni $z$ ni y quieren formar parte de él, entonces $C$ no es elegible. Supongamos el conjunto $D:\{v, w, x, z, y\}$-todo conjunto es un subconjunto de sí mismo-, en donde $v$ y w no quieren formar parte de él, pero $x, y, z$, si quieren. Entonces $D$ es elegible. ¿Cuál es el criterio para elegir entre B y D, ambos elegibles? Es obvio que, si sólo disponemos del criterio de la voluntad de los miembros de cada subconjunto, el problema Matrioska es irresoluble.

Y aquí hay una diferencia con el derecho de asociación, porque el derecho de asociación no exige que tengamos que elegir entre los diversos conjuntos. Podemos considerarlos todos elegibles, si tienen una mayoría. Podemos exigir, como el derecho de asociación parece requerir, la unanimidad. Pero no hay dificultad alguna en que haya unanimidad para elegir un subconjunto determinado y también otro. Nada prohíbe asociarse a diversos grupos, algunos de los cuales son subconjuntos de los otros. Sin embargo, en el caso de la secesión, que involucra el poder soberano sobre un territorio, ello es imposible. No hay razones, dependientes únicamente de la voluntad de los miembros, para elegir entre el conjunto $B$ y el conjunto $D$.

En acertadas palabras de Beitz (1979, p. 110):

La crucial diferencia, claro está, es que las asociaciones voluntarias no son grupos territoriales: usualmente no tienen que vivir juntos en un territorio separado o privar a otros del territorio en el que habitaban previamente. Mientras la creación de una 
asociación voluntaria conlleva la partición de algunos grupos de personas, no conlleva la partición de territorio alguno.

Una consecuencia de todo ello está bien captada por Ferrajoli (2007, p. 575) al referirse al derecho de autodeterminación externa:

No es concebible, como derecho universal y por ello fundamental, ni siquiera en el plano teórico, al ser en este sentido lógicamente inconsistente y prácticamente autodestructivo. En la minoría que protagoniza la secesión habrá siempre otra minoría que, a su vez, querrá llevarla a cabo frente a la vieja minoría convertida en mayoría.

Vilajosana (2020, p. 386) es bien consciente de esta objeción:

Una de las objeciones recurrentes de los contrarios al derecho a decidir es que hay dificultades para la determinación del demos, es decir, de cuál es el colectivo relevante a la hora de ser consultado. Así se dirá que si los catalanes tienen ese derecho, qué impide que lo tengan los habitantes del área metropolitana de Barcelona; [...] Dicho en términos generales, nada habría que justificase que una clase determinada de individuos sea la relevante para ser la titular de ese derecho, ya que podría serlo cualquier subclase de la misma.

Y esta es su respuesta a esta objeción (Vilajosana, 2020, p. 386):

Frente a este argumento cabe oponer lo siguiente. Para empezar, o el argumento es plausible o no lo es. Si no lo es, es decir, si no se considera que la conclusión es absurda, no hace falta que le dediquemos más tiempo. Si, en cambio, se supone que es plausible, entonces habrá que admitir que se aplica no solo a la clase de los catalanes sino a cualquier otra clase de ciudadanos. Así, la misma reducción al absurdo se produciría respecto a España. ¿Por qué los españoles pueden decidir su futuro político y no cualquier subclase de ciudadanos, entre los cuales por cierto la de los catalanes? Ante este contraargumento, ya sólo queda refugiarse en la defensa de lo existente.

En un punto lleva toda la razón Vilajosana: si el contraargumento Matrioska vale, vale contra todas las comunidades, también contra la que coincide con la estatal, ya decía que todo conjunto es un subconjunto de sí mismo. Sin embargo, ello no invalida el argumento, sólo dice que el derecho a decidir no es un modo adecuado de formular una pretensión de legitimidad, porque no determina nunca de manera unívoca su titular. Ahora bien, y este es el punto final de Vilajosana, ¿por qué hemos de defender el marco existente?

En primer lugar, la defensa del marco existente no se deriva de la objeción Matrioska, lo único que dice la objeción Matrioska es que concebir el derecho a la secesión como una extensión del derecho de asociación es un error, porque el derecho de asociación no comporta la reivindicación de la soberanía exclusiva y excluyente sobre un territorio y, entonces, no tiene el problema de la indeterminación de la elección de un subconjunto. Uno puede ser socio del Barça, miembro de Amnistía Internacional, miembro del club de lectura de la biblioteca del barrio, etc. No hay que elegir entre estas pertenencias. Nada más, pero nada menos.

En segundo lugar, aunque las fronteras son arbitrarias sin ninguna duda, hay argumentos para sostener que, como dice Dworkin (2011, p. 382): «las fronteras generadas por accidentes de la historia se mantienen por defecto». Esto no es cometer, como sugiere Vilajosana (2020, p. 387) ninguna falacia naturalista. Consideremos una analogía: la justificación de la familia (vid. Rawls, 1999, p. 488) y la sujeción de los hijos menores de edad a sus padres. Es obvio que el hecho de nacer de unos padres determinados es moralmente irrelevante y arbitrario. Sin 
embargo, es susceptible de generar deberes y derechos a los padres y a los hijos. Hay razones, claro, para retirar la patria potestad a los padres cuando desatienden gravemente sus deberes. Pero son razones de reparación, son razones como «remedios». Por dicha razón, siguiendo con la analogía, el derecho unilateral a la secesión sólo emerge como consecuencia de injusticias previas, como una reparación, como quiere el autor que más ha pensado sobre todo ello contemporáneamente, me refiero a Buchanan (2004, p. 2017). Es decir, completando la frase de Dworkin, «en ausencia de causas justas, las fronteras se mantienen por defecto». No hay falacia naturalista alguna, hay una doctrina que exige determinados requisitos para conceder un derecho a la secesión unilateral, y los exige por razones normativas. En cambio, la vía del derecho a decidir no está en condiciones de ofrecer lo que promete, puesto que carece de recursos para delimitar el titular de ese derecho de manera unívoca.

Aunque Vilajosana nunca usa el otro argumento relevante en la teoría contemporánea a favor del derecho a la secesión unilateral, que es el argumento nacionalista ( vid, por todos Margalit-Raz, 1990 y, entre nosotros, Payero López, 2016 y Torbisco, 2017), al menos en una ocasión usa esta idea también (Vilajosana, 2020, p. 387), cuando se refiere a las minorías dice: "en este caso una minoría con contornos territoriales definidos y con lengua, cultura e instituciones propias». Entonces, tal vez, podría argumentarse que el problema de la vía plebiscitaria podría resolverse mediante la adición de la vía nacionalista. Aunque aquí no puedo argumentarlo sin alargar en demasía este comentario, esto no resuelve el problema puesto que la vía plebiscitaria precisa de un modo externo a su razonamiento de delimitar el demos titular del derecho, y el nacionalismo no está en condiciones de proporcionárselo (Moreso ms.). Dicho ahora muy brevemente, no está en condiciones de proporcionárselo porque no hay modo de delimitar con precisión cuál es el territorio, y la secesión está intrínsecamente vinculada a la soberanía sobre un territorio, en donde habita una y sólo una comunidad cultural. Aprovechándome de una idea de Gowans (2019), elaborada para un tema en la vecindad de éste, el del relativismo cultural, el autor nos dice que las culturas no son como un cuadro del gran pintor Piet Mondrian, con límites precisos -cómo sí lo son los Estados-, sino que son más bien como un cuadro del no menos grande Jackson Pollock, en los cuales todo fluye y no hay forma de introducir límites precisos.

\section{A modo de conclusión: el poder y el derecho}

Cuando abandonemos el confinamiento al que la pandemia del COVID-19 nos ha sometido, será el momento de regresar al diálogo en el asunto catalán. Un diálogo en el cual, en mi opinión, el gobierno de España debe contemplar, de entrada, todas las posibilidades para el futuro de Cataluña, y el gobierno de la Generalitat debe abandonar la unilateralidad y recordar, como bien sostiene Vilajosana, que la unilateralidad no cabe en la Constitución, ni está justificada, la unilateralidad es, en este supuesto, ilegítima. Como he dicho en otra ocasión (Moreso, 2018), el diálogo no ha sido posible por el abandono de la política por el gobierno de Madrid, y el menosprecio del derecho por parte del gobierno catalán. Y como escribió Norberto Bobbio una vez (Bobbio, 1982): «ll potere senza diritto è cieco, ma il diritto senza potere è vuoto».

Si lo hacemos de este modo, y así desvelo el propósito con el que he seleccionado el pasaje del Libro de los Jueces que figura en el atrio de estos comentarios, seremos capaces tal vez de no exigir shibolets, que son una especie de santo y seña, a nuestros interlocutores y de prescindir de los shibolets, que son también nuestros dogmas, que nublan nuestra visión adecuada de las cosas. 
Bibliografía

Alchourrón, C. y Bulygin, E. (1971), Normative Systems. New York-Wien, Estados Unido: Springer.

Barceló, M. (2017). El derecho a decidir como instrumento constitucional para la canalización de problemas territoriales. Fundamentos: Cuadernos monográficos de teoría del estado, derecho público e historia constitucional, 9, pp. 361-391.

Bayona, A. (2019). No todo vale. La mirada de un jurista a las entrañas del 'procés'. Barcelona, España: Península.

Beitz, Ch. (1979). Political Theory and International Relations. Princeton, Estados Unidos: Princeton University Press.

Beran, H. (1984). A Liberal Theory of Secession. Political Studies, 32, pp. 21-31.

Beran, H. (1995). A Democratic Theory of Political Self-Determination for a New World Order. En P. B. Lehning (ed.). Theories of Secession (pp. 33-61). Londres, Reino Unido: Routledge.

Bobbio, N. (1982). Il potere e il diritto. Nuova antología, A. 117, vol. 549, fasc. 2142, pp. $68-80$.

Buchanan, A. ( 2004). Justice, Legitimacy, and Self-Determination: Moral Foundations for International Law. Oxford, Reino Unido: Oxford University Press.

Buchanan. A. (2017). Secession. The Stanford Encyclopedia of Philosophy (Fall 2017 Edition, Edward N. Zalta [ed.]). Recuperado de https://plato.stanford.edu/archives/fall2017/entries/secession/.

Cassese, A. (1995). Self-Determination of Peoples: A Legal Reappraisal. Cambridge, Reino Unido: Cambridge University Press.

Dworkin, R. (2011). Justice for Hedgehogs. Cambridge, Mass., Estados Unidos: Harvard University Press.

Ferrajoli, L. (2007). Principia luris. Teoria del diritto e della democracia. Vol. 2. RomaBari, Italia: Laterza.

Ferreres, V. (2014). The Spanish Constitutional Court Confronts Catalonia's Right to Decide (Comment on the Judgment 42/2014). European Constitutional Law Review, 10, pp. 571-590.

Ferreres, V. (2016). Cataluña y el derecho a decidir. Teoría y Realidad Constitucional, 37, pp. 461-475.

Fossas, E. (2014). Interpretar la política: Comentario a la STC 42/2014, de 25 de marzo, sobre la Declaración de soberanía y el derecho a decidir del pueblo de Cataluña. Revista Española de Derecho Constitucional, 101, pp. 287-289.

Hohfeld, W.N, (1919). Fundamental Legal Conception. New Haven, Estados Unidos: Yale University Press.

Gowans, C. (2019). Moral Relativism. The Stanford Encyclopedia of Philosophy (Summer 2019 Edition, Edward N. Zalta [ed.]). Recuperado de https://plato.stanford.edu/archives/sum2019/entries/moral-relativism/.

López, J. (2017). El derecho a decidir. La vía catalana. Tafalla, España: Txalaparta.

Margalit, A. y Raz, J. (1990). National Self- Determination. Journal of Philosophy, 87(9), pp. 439-461.

Moreso, J.J. (2018). Lecciones canadienses: sobre democracia, constitucionalismo y federalismo. En B. Pendás (dir.), E, González, R. Rubio (coords.), España Constitucional (1978-2018). Trayectorias y Perspectivas, Tomo II (pp. 16571673). Madrid, España: Centro de Estudios Políticos y Constitucionales.

Moreso, J.J. (s.f.). De secessione. Lo escondites de la vía catalana. Inédito. Recuperado de https://upf.academia.edu/Jos\%C3\%A9JuanMoreso

Payero López, L. (2016). El derecho de autodeterminación en España: Breve explicación para extranjeros estupefactos y nacionales incautos. Revista de Estudios Autonómicos y Federales, 23, pp. 46-79.

Philpott, D. (1995). A Defense of Self-Determination. Ethics, 105, pp. 352-85. 
Queralt, A. y Martí, J.L. (18 diciembre, 2017). Un referéndum para Cataluña. Agenda Pública, El País. Recuperado de http://agendapublica.elpais.com/un-referendumpara-catalunal

Rawls, J. (1999). A Theory of Justice. Cambridge, Mass. Estados Unidos: Harvard University Press.

Rubio Llorente, F. (8 octubre, 2012): Un referéndum para Cataluña. El País. https://elpais.com/elpais/2012/10/03/opinion/1349256731 659435.html

Torbisco, N. (2017). National Minorities, Self-determination and Human Rights: A Critique of the Dominant Paradigms in the Catalan Debat. En P. A. Kraus, J. Vergés (eds.). The Catalan Process, Sovereignty, Self-Determination and Democracy in the 21st Century (pp. 199-232). Barcelona, España: Generalitat de Catalunya-Institut d'Estudis de l'Autogovern.

Tornos, J. (2015). De Escocia a Cataluña. Referéndum y reforma constitucional. Madrid, España: lustel.

Vilajosana, J. M. (2014a). Principi democràtic i justificació constitucional del dret de decidir. Revista d'estudis autonòmics i federals, 19, pp. 178-210.

Vilajosana; J.M. (2014b). The democratic principle and constitutional justification of the right to decide. Catalan Social Sciences Review, 4, pp 57-80.

Vilajosana, J.M. (2019). Laboratorio Cataluña. Legalidad, legitimidad y eficacia en el proceso político catalán. En I. Lasgabaster (coord.), Crisis institucional y democracia (A propósito de Cataluña) (pp. 75-99). Valencia, España: Tirant lo Blanch.

Vilajosana, J.M. (2020). Democracia y derecho a decidir. Eunomía. Revista en Cultura de la legalidad, 18, pp. 375-391.

Von Wright, G.H. (1963). Norm and Action. A Logical Enquiry. Londres, Reino Unido: Routledge \& Kegan Paul.

Waluchow, W.J. (2007). A Common Law Theory of Judicial Review: The Living Tree. Cambridge, Reino Unido: Cambridge University Press.

Wellman, C. (1995). A Defense of Secession and Political Self-Determination. Philosophy \& Public Affairs, 24, pp. 142-171. 